\title{
Multisensor Distributed Information Fusion White Noise Wiener Deconvolution Estimator
}

\author{
Yun $\mathrm{Li}^{1,2}$, Ming Zhao ${ }^{1}$, Gang $\mathrm{Hao}^{2} *$, Junling $\mathrm{Li}^{1}$ and Hao $\mathrm{Jin}^{1}$ \\ 1 School of Computer and Information Engineering \\ Harbin University of Commerce \\ HeiLongJiang, Harbin, 150001, China \\ $2 *$ Electronic Engineering Institute \\ Heilongjiang University \\ Heilongjiang, Harbin, 150080, China \\ liyunhd@hrbcu.edu.cn,haogang@hlju.edu.cn
}

\begin{abstract}
Multisensor distributed information fusion white noise wiener deconvolution estimator is presented in this paper. The algorithm is using the modern time series analysis method and white noise estimator under the linear minimum variance optimal fusion criterion. Gevers-Wouters $(G-W)$ algorithm are also used in this paper. This paper presents information fusion algorithm including scalar weighted and covariance intersection fusion. The algorithm analyzes the relationship between the accuracy and the computation of the two fusion algorithm. The formula of optimal weighting coefficients is given. Compared with the single sensor case, the accuracy of the fused filter is greatly improved. It can be applied to signal processing in oil seismic exploration, communication and other fields. A simulation example for information fusion BernoulliGaussian white noise deconvolution filter shows its correctness and effectiveness.
\end{abstract}

Keywords: Distributed information fusion, Reflection seismology, Wiener deconvolution estimator, White noise estimator

\section{Introduction}

The important application background for white noise estimator is the signal processing in oil seismic exploration[1]. The sensor is essentially a dynamic system. Its input is white noise reflection sequence and its output is the received signal. The problem is to estimate the white noise input signal by the sensor output signal with measurement noise, this kind of problem is called deconvolution[2]. It is important to judge whether the oil is existed and the oil field geometry. In this background, Mendel has proposed a white noise estimation theory by using the Kalman estimator, and given the application in oil seismic exploration. But the theory has shortcomings and limitations. It only gives the optimal input white noise estimators, and do not give the optimal measurement white noise estimators. So its application is limited to deconvolution, and it cannot be used to solve the problem of signal and state estimation.

Since 1989, Professor Deng Zili put forward the modern time series analysis method[3]. This method is different from the Kalman filtering method and Wiener estimator method, and it is a new time-domain method. It takes ARMA model as the basic tool. The key technologies include (1) The method uses the ARMA innovation model; (2) The method obtains non recursive state expressions of the white noise and signal; (3) The method need to calculate the white noise estimators and measurement predictor; (4) The method involves the transformation of the state space model and ARMA time series model; (5) The method is based on projection theory.

*Corresponding author: Gang Hao 
The characteristic of this method is: it avoids solving the Riccati equation compared with the Kalman filtering method, so it is simple. But this method is limited to the constant (time invariant) systems. Compared with the classical Wiener filtering method, this method avoids the partial fraction expansion of the transfer function and can handle the multidimensional nonstationary signal. Compared with the polynomial method, this method avoids solving the Diophantine equation.

Classical optimal filtering theory is based on the single sensor system. The multisensor information fusion filtering theory is formed by mutual penetration, intersect of the classical optimal filtering theory and multisensor information fusion. It mainly studies the multisensor information fusion state (or signal) estimation problems and self-tuning information fusion state (or signal) estimation problem. One of the most important applications of multisensor information fusion filtering is target tracking. In order to improve tracking accuracy of the moving target state, the multisensor is used. The purposes of the information fusion are combining the local state estimation (observation equations) to obtain a fused estimation (observation equations). The basic requirements for fusion design are that the accuracy of information fusion is higher than the local estimator. Its field includes guidance, GPS, defense, medical, integrated navigation, target tracking, robot technology, communications and signal processing[4] [6]. Distributed fusion Kalman filtering under linear minimum variance rules has four information fusion algorithms: the matrix weighted, scalar weighted and diagonal matrix weighted. Compared with the centralized fuser, the distributed fuser can reduce the calculation burden and are more flexible and reliable. The distributed fusion estimation needs to calculate the cross-covariances of local estimate[7] [10]. However, in many theoretical and application problems, the cross-covariances are unknown, or it is very difficult to compute the cross-covariances, or can't calculate the cross-covariances. If the crosscovariances are neglected, it will lead the increase of the variance of the local estimator error, even the divergence of the estimator.

In order to overcome the disadvantages and limitations, Jeffrey K. Uhlman proposed the covariance intersection information fusion method. It is also a distributed information fusion method, and it completely avoids the unknown cross-covariance identification and calculation. It can deal with the fusion estimation problem for the system with unknown covariance. Compared to the other fusion estimator, covariance intersection fusion algorithm has the advantages as follows[11] [14]:

(1) This algorithm avoids computing the covariance, so it can reduce the computational burden.

(2) The algorithm can solve the multisensor fusion problem with unknown covariance.

(3) The precision of the covariance intersection fusion is better than the local estimator accuracy. Its accuracy is close to the optimal matrix fusion Kalman estimator in many cases, so it has excellent performance.

(4) The algorithm can be used to fusion estimator for nonlinear systems.

In this paper, white noise Wiener deconvolution estimator is weighted by scalars and covariance intersection fusion for discrete time stochastic linear systems. The algorithm is under the linear minimum variance sense, and the optimal information fusion criterion includes scalars and covariance intersection fusion. The accuracy of scalars is better than the one of covariance intersection fusion. But the computational burden is on the contrary. Fusion estimator weighted by scalars has a large computational burden, and weighted by covariance intersection fusion with small computational burden, so it is suitable for realtime applications. A simulation example for the typical track system with 2-sensor shows the correctness and validity of training. And simulation results show no significant difference between the two kinds of distributed fusion algorithm. 
The main structure of this paper is as follows: Problem formulation is given in Section 2. Local optimal white noise Wiener deconvolution estimator is obtained in Section 3. In Section 4 distributed information fusion optimal white noise Wiener deconvolution estimator is presented. A simulation example with 2-sensor is given in Section 5. In Section 6 the conclusions of this paper are given.

\section{Problem Formulation}

Consider the multisensor discrete-time linear time-invariant single channel deconvolution system

$$
\begin{gathered}
x_{i}(t+1)=\widetilde{J}_{i} x_{i}(t)+\aleph_{i} w(t) \\
y_{i}(t)=\mathfrak{R}_{i} x_{i}(t)+v_{i}(t), i=1, \cdots, l
\end{gathered}
$$

where $l$ is the number of the sensor, $l \geq 2$. And $x_{i}(t) \in R^{n}$ is the state of the $i$ th sensor, the scalar $y_{i}(t) \in R^{m i}$ is the measurement (output) of the $i$ th sensor subsystem, $v_{i}(t) \in R^{m i}$ is the measurement noise of the $i$ th sensor subsystem, $w(t) \in R^{r}$ is the input noise, $\mathfrak{J}_{i}, \aleph_{i}, \mathfrak{R}_{i}$ is the known constant matrix.

Assumption $1 w(t) \in R^{r}$ and $v_{i}(t) \in R^{m i}, i=1, \cdots, L$ are independence white noises with zero mean and covariance are $Q_{w}$ and $Q_{v i}$ individually.

$$
\mathrm{E}\left\{\left[\begin{array}{l}
w(t) \\
v_{i}(t)
\end{array}\right]\left[\begin{array}{ll}
w(k) & v_{i}(k)
\end{array}\right]\right\}=\left[\begin{array}{cc}
Q_{w} & 0 \\
0 & Q_{v i}
\end{array}\right] \delta_{t k}
$$

where $\mathrm{E}$ is the mathematical expectation, $\delta_{t t}=1, \delta_{t k}=0(t \neq k)$.

Assumption $2\left(\mathfrak{I}_{i}, \mathfrak{R}_{i}\right)$ is the completely observable pairs, $\left(\mathfrak{I}_{i}, \mathfrak{\aleph}_{i}\right)$ is the completely controllable pairs.

The aim is to find the local optimal deconvolution estimator $\hat{w}_{i}(t \mid t+N), i=1,2 \cdots l$ and the optimal distributed fusion deconvolution estimator $\hat{w}_{0}(t \mid t+N)$ based on the measurement $\left.\left(y_{i}(t+N)\right), y_{i}(t+N-1), \cdots\right), N \geq 0$. For $N=0, N>0$ or $N<0$, we referred to as white noise deconvolution filter, smooth or predictor.

\section{Local Optimal White Noise Wiener Deconvolution Estimator}

Theorem 1 For the system (1) and (2) under the Assumption 1-2, the asymptotically stability local optimal white noise wiener deconvolution estimator $\hat{w}_{i}(t \mid t+N)$ of the $i$ th sensor is as follows

$$
\begin{gathered}
D_{i}\left(q^{-1}\right) \hat{w}_{i}(t \mid t+N)=\lambda_{N W}^{(i)}\left(q^{-1}\right) B_{i}\left(q^{-1}\right) y_{i}(t+N), i=1,2, \cdots, l \\
\lambda_{N W}^{(i)}\left(q^{-1}\right)=\frac{\sigma_{w}^{2}}{\sigma_{\varepsilon_{i}}^{2}} \sum_{j=0}^{N} \hbar_{j}^{(i)} q^{j-N}, i=1,2, \cdots, l, N \geq 0
\end{gathered}
$$

The coefficient $\hbar_{j}^{(i)}$ can be recursive calculated as

$$
\hbar_{j}^{(i)}=-d_{1}^{(i)} \hbar_{j-1}^{(i)}-\cdots-d_{n_{d i}}^{(i)} \hbar_{j-n_{d i}}^{(i)}+\vartheta_{j}^{(i)}
$$

where letting $\hbar_{j}^{(i)}=0(j<0), \vartheta_{j}^{(i)}=0\left(j>n_{g_{i}}\right)$.

And the innovation estimator is

$$
\hat{w}_{i}(t \mid t+N)=\frac{\sigma_{w}^{2}}{\sigma_{\varepsilon_{i}}^{2}} \sum_{j=0}^{N} \hbar_{j}^{(i)} \varepsilon_{i}(t+j), i=1,2, \cdots, l
$$

Proof: From (1) and (2) having

$$
y_{i}(t)=\mathfrak{R}_{i}\left(I_{n}-q^{-1} \mathfrak{J}_{i}\right)^{-1} \aleph_{i} q^{-1} w(t)+v_{i}(t)
$$


where $q^{-1}$ is the unit delay operator. This leads to

$$
\varpi_{i}\left(q^{-1}\right) y_{i}(t)=\vartheta_{i}\left(q^{-1}\right) w(t)+\varpi_{i}\left(q^{-1}\right) v_{i}(t)
$$

and polynomial $\varpi_{i}\left(q^{-1}\right)$ and $\vartheta_{i}\left(q^{-1}\right)$ are defined as

$$
\begin{gathered}
\varpi_{i}\left(q^{-1}\right)=\operatorname{det}\left(I_{n}-q^{-1} \mathfrak{\Im}_{i}\right) \\
\vartheta_{i}\left(q^{-1}\right)=\mathfrak{R}_{i} \operatorname{adj}\left(I_{n}-q^{-1} \mathfrak{J}_{i}\right) \aleph_{i} q^{-1}
\end{gathered}
$$

They can be written as $X_{i}\left(q^{-1}\right)=x_{0}^{(i)}+x_{1}^{(i)} q^{-1}+\cdots+x_{n_{x i}}^{(i)} q^{-n_{n_{i j}}}, \varpi_{0}^{(i)}=0, \vartheta_{0}^{(i)}=0$.

The ARMA innovation model is as follows from (9)

$$
\varpi_{i}\left(q^{-1}\right) y_{i}(t)=\alpha_{i}\left(q^{-1}\right) \varepsilon_{i}(t)
$$

where $\alpha_{i}\left(q^{-1}\right)$ is stable, $\alpha_{0}^{(i)}=1$, and innovation $\varepsilon_{i}(t)$ is white noises with zero mean and covariance are $Q_{\varepsilon i}$, and having

$$
\alpha_{i}\left(q^{-1}\right) \varepsilon_{i}(t)=\vartheta_{i}\left(q^{-1}\right) w(t)+\varpi_{i}\left(q^{-1}\right) v_{i}(t)
$$

$\alpha_{i}\left(q^{-1}\right)$ and $Q_{\varepsilon i}$ can be computed by Gevers-Wouters ${ }^{[3]}$.

The optimal white noise estimator is as (7) in literature [3], and $\hbar_{j}^{(i)}$ is defined as(6). So we have

$$
\hat{w}_{i}(t \mid t+N)=\lambda_{N W}^{(i)}\left(q^{-1}\right) \varepsilon_{i}(t+N)
$$

$\lambda_{N W}^{(i)}\left(q^{-1}\right)$ is defined as $(5)$.

Substitutting (12) into (14), so (4) is obtained. Because of the stability of $\alpha_{i}\left(q^{-1}\right)$ leads to (4) is asymptotically stable.

The proof is completed.

Theorem 2 For the system (1) and (2) under the Assumption 1-2, the local steadystate optimal estimation error covariance $P_{i}(N)=\mathrm{E}\left[\tilde{w}_{i}^{2}(t \mid t+N)\right]$ is given as

$$
\begin{gathered}
P_{i}(N)=Q_{w}\left[1-\frac{Q_{w}}{Q_{\varepsilon i}} \sum_{j=0}^{N}\left(\hbar_{j}^{(i)}\right)^{2}\right], \quad i=1,2 \cdots l, \quad N \geq 0 \\
P_{i}(N)=Q_{w}, \quad N<0
\end{gathered}
$$

and the cross covariance $P_{i j}(N)=\mathrm{E}\left[\tilde{w}_{i}(t \mid t+N) \tilde{w}_{j}(t \mid t+N)\right](i \neq j)$ between any two local sensor is computed by

$$
\begin{gathered}
P_{i j}(N)=Q_{w}-\frac{\left(Q_{w}^{2}\right)^{2}}{Q_{\varepsilon_{i}}} \sum_{r=0}^{N}\left(\hbar_{r}^{(i)}\right)^{2}-\frac{\left(Q_{w}\right)^{2}}{Q_{\varepsilon_{j}}} \sum_{s=0}^{N}\left(\hbar_{s}^{(j)}\right)^{2}+\frac{\left(Q_{w}\right)^{2}}{Q_{\varepsilon_{i}} Q_{\varepsilon_{j}}} \sum_{r=0}^{N} \sum_{s=0}^{N} \hbar_{r}^{(i)} \hbar_{s}^{(j)} \Theta_{i j}(r, s), N \geq 0 \\
\Theta_{i j}(r, s)=\sum_{k=\max (0, r-s)}^{\infty} \hbar_{k}^{(i)} \hbar_{s-r+k}^{(j)} \sigma_{w}^{2} \\
P_{i j}(N)=Q_{w}, N<0
\end{gathered}
$$

Proof: By the (13) with expansion ${ }^{[3]}$ :

$$
\varepsilon_{i}(t)=\frac{\varpi_{i}\left(q^{-1}\right)}{\alpha_{i}\left(q^{-1}\right)} w(t)+\frac{\vartheta_{i}\left(q^{-1}\right)}{\alpha_{i}\left(q^{-1}\right)} v_{i}(t)
$$

By (7) and (20), so (15), (17) and (18) are obtained.

When $N<0$, the above derivation process and $\hbar_{0}^{(i)}=0$, so (16) and (19) are established. 
The proof is completed.

\section{Distributed Information Fusion Optimal White Noise Wiener Deconvolution Estimator}

Lemma 1 For the system (1) and (2), under the same conditions, the optimal fused white noise Wiener deconvolution estimator $\hat{w}_{0}(t \mid t)$ weighted by scalars is g

$$
\hat{w}_{0}(t \mid t)=\sum_{i=1}^{L} \wp_{i}(t) \hat{w}_{i}(t \mid t)
$$

Under the linear minimum variance optimal information fusion criterion which minimize the performance index, the optimal weighting coefficients $\wp_{i}(t), i=1,2, \cdots, M$ are given by

$$
\left[\wp_{1}(t), \cdots, \wp_{l}(t)\right]=\frac{e^{\mathrm{T}} P_{\mathrm{tr}}^{-1}}{e^{\mathrm{T}} P_{\mathrm{tr}}^{-1} e}
$$

where we define the $M \times M$ matrix $P_{\mathrm{tr}}=\left(\operatorname{tr} P_{i j}\right)_{M \times M}, i, j=1,2, \cdots, M$, and $P_{i j}$ can be calculated by Theorem 2 , and $M \times 1$ row vector $e=\left[\begin{array}{lll}1 & \cdots & 1\end{array}\right]^{\mathrm{T}}$.

The optimal fused variance matrix is given as

$$
\boldsymbol{P}_{0}(N)=\sum_{j, i=1}^{M} \wp_{i}(k) \wp_{j}(k) \boldsymbol{P}_{j i}(N)
$$

and

$$
\operatorname{tr} \boldsymbol{P}_{0} \leq \operatorname{tr} \boldsymbol{P}_{j}, j=1,2, \cdots, M
$$

Lemma 2 For the system (1) and (2), under the same conditions, when the variance of $\mathrm{P}_{1}$ and $\mathrm{P}_{2}$ are known, but the cross covariance $\mathrm{P}_{12}$ is unknown, using the covariance intersection (CI) fusion method, this paper proposes a suboptimal fusion Kalman estimators is as follows ${ }^{[16]}$ :

$$
\hat{w}_{C I}(t \mid t)=\sum_{i=1}^{L} \beta_{i}(t) \hat{w}_{i}(t \mid t)
$$

Fusion weight is calculated as follows

$$
\beta_{i}(t)=\lambda_{i}(t)\left(\sum_{i=1}^{L} \lambda_{i}(t) P_{i}^{-1}(t \mid t)\right)^{-1} P_{i}^{-1}(t \mid t)
$$

where

$$
\lambda_{i}(t)=\frac{\operatorname{tr}\left(P_{i}^{-1}(t \mid t)\right)}{\sum_{i=1}^{L} \operatorname{tr}\left(P_{i}^{-1}(t \mid t)\right)}, 0 \leq \lambda_{i}(t) \leq 1, \sum_{i=1}^{L} \lambda_{i}(t)=1
$$

It is proved in document [3] that the accuracy of above two kinds of weighted fusion estimator from high to low is scalars and covariance intersection fusion. But the computational burden is on the contrary, fusion estimator weighted by scalars has a large computational burden. And covariance intersection fusion avoids solving cross-covariance matrices. So it has the minimal computational burden, and it is suitable for real-time applications. 


\section{Simulation Example}

Consider the multisensor single channel deconvolution system

$$
\begin{gathered}
x_{i}(t+1)=\mathfrak{J}_{i} x_{i}(t)+\aleph_{i} w(t) \\
y_{i}(t)=\mathfrak{R}_{i} x(t)+v_{i}(t), i=1,2
\end{gathered}
$$

where $\mathfrak{I}_{1}=\left[\begin{array}{ll}-0.5 & 1 \\ -0.6 & 0\end{array}\right], \mathfrak{I}_{2}=\left[\begin{array}{ll}-0.4 & 1 \\ -0.5 & 0\end{array}\right], \aleph_{1}=\left[\begin{array}{c}1 \\ 0.2\end{array}\right], \aleph_{2}=\left[\begin{array}{c}1 \\ 0.6\end{array}\right], \mathfrak{R}_{1}=\left[\begin{array}{ll}1 & 0\end{array}\right], \mathfrak{R}_{2}=\left[\begin{array}{ll}2 & 0\end{array}\right]$. $w(t)=b(t) g(t)$ is Bernoulli-Gaussian white noise, where $b(t)$ is Bernoulli white noise and its value is 1 and 0 . Further the probability for $b(t)$ is: $\mathrm{P}(b(t)=1)=\lambda, \mathrm{P}(b(t)=0)=1-\lambda$, $\lambda=0.3$. While $g(t)$ is white noise with zero mean and covariance $\sigma_{g}^{2}=1$ which is independent of $b(t)$. This leads to $\sigma_{w}^{2}=\lambda \sigma_{g}^{2}=0.3$. And $v_{i}(t)$ is white noise with zero mean which is independent of $w(t)$. Further its covariance is $\sigma_{v 1}^{2}=0.01, \sigma_{v 2}^{2}=0.02$ individually. The problem is to obtain the local optimal deconvolution smooth $\hat{w}_{i}(t \mid t+2)$ and the optimal distributed fusion deconvolution smooth $\hat{w}_{0}(t \mid t+2)$.

In the simulation the ARMA model parameters is as follows when $\mathrm{t}=300$ :

$$
\begin{gathered}
d_{1}^{(1)}=0.2097, d_{2}^{(1)}=0.0019, \sigma_{\varepsilon 1}^{2}=0.3109 \\
d_{1}^{(2)}=0.5841, d_{2}^{(2)}=0.0031, \sigma_{\varepsilon 2}^{2}=0.3215
\end{gathered}
$$

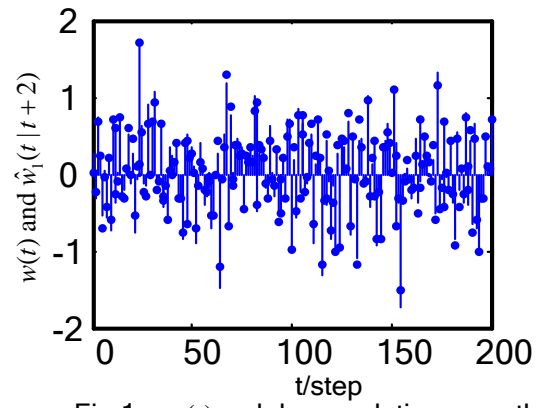

Fig.1 $w(t)$ and deconvolution smooth $\hat{w}_{1}(t \mid t+2)$ of the first sensor

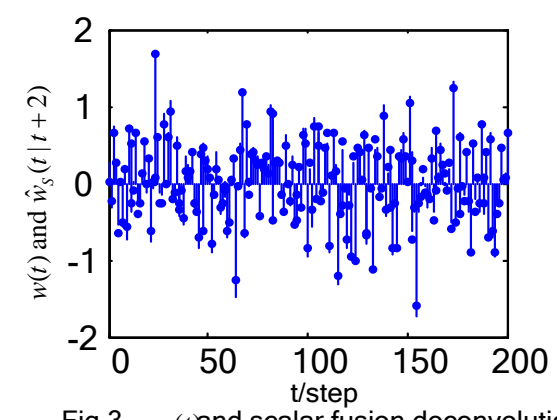

Fig.3 $w(t$ ) and scalar fusion deconvolution $\hat{w}_{S}(t \mid t+2)$ smooth

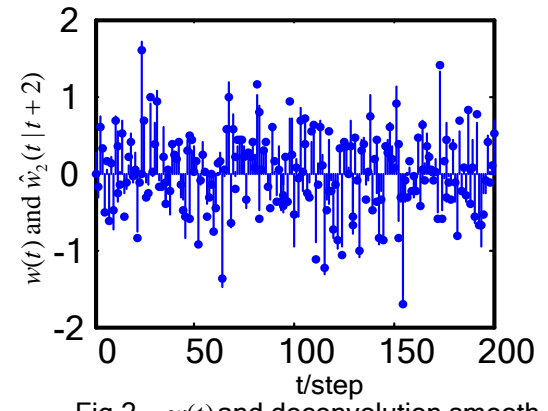

Fig.2 $w(t)$ and deconvolution smooth $\hat{w}_{2}(t \mid t+2)$ of the second sensor

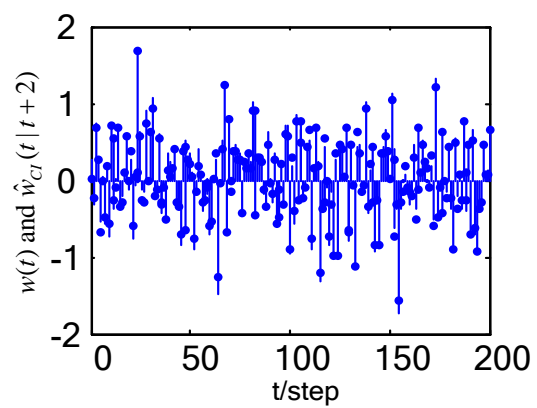

Fig.4 $w(t)$ and $\mathrm{Cl}$ fusion deconvolution $\hat{w}_{C I}(t \mid t+2)$ smooth 


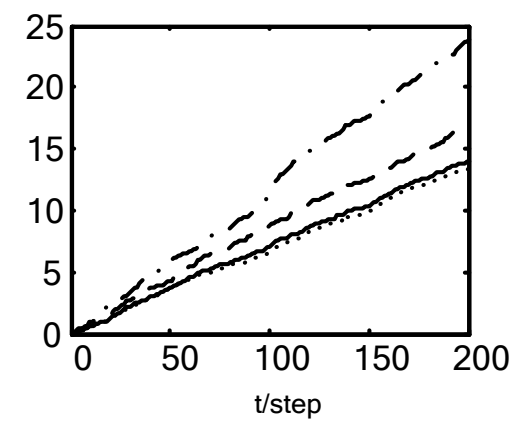

Fig. 5 The curves of the sum of absolute error curve for local and fusion white noise smooth

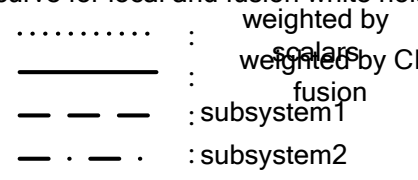

The simulation results are shown in Fig.1-Fig.5. Figure 1 and 2 gives the local optimal deconvolution estimator, and the fusion smooth weighted by scalars and the covariance intersection (CI) fusion are shown in Figure 3 and 4. Where the solid line represents true value $w(t)$, and dot ordinate represents estimator value $\hat{w}(t \mid t+2)$. We can see from the chart that the accuracy of the fusion smooth is higher than that of each local smooth. Figure 5 is the absolute error curve for the local and fusion white noise smooth weighted by scalars and the covariance intersection. Simulation results show no significant difference between the two distributed fusion algorithm. But the fusion estimator weighted by scalars has a large computational burden. And covariance intersection fusion avoids solving cross-covariance matrices. So it has the minimal computational burden, and it is suitable for real-time applications.

\section{Conclusions}

Using the modern time series analysis method and white noise estimation theory in this paper, under the linear minimum variance optimal information fusion criterion, and the multisensor optimal information fusion white noise deconvolution estimator is presented. The algorithm presented in this paper has many advantages.

(1) Information fusion rule adopted in this paper is weighted by scalars and covariance intersection fusion. The estimation accuracy for the system is greatly improved compared with the single local sensor. The accuracy of above two kinds of weighted fusion estimator is from high to low. But the computational burden is on the contrary. Fusion estimator weighted by scalars has a large computational burden, and covariance intersection fusion with minimal computational burden, and it is suitable for real-time applications. The simulation example shows its validity.

(2) The advantages of this method can design self-tuning information fusion white noise deconvolution estimator with unknown model parameters and noise variance based on the online identification of ARMA innovation model.

(3) In this paper, the proposed algorithm can be applied to signal processing in oil seismic exploration, communication and other fields.

\section{Acknowledgements}

This work is supported by Key Laboratory of Electronics Engineering, College of Heilongjiang Province, (Heilongjiang University), P. R. China, by science and technology research foundation of Heilongjiang education department under Grant 
12531159, by the Natural Science Foundation of Heilongjiang Province of China (Grant Nos. F201426), by Harbin University of Commerce Youth Fund.

\section{References}

[1] C. Y. Han and Y. Zhang, "Suboptimal white noise estimators for discrete time systems with random delays", Signal Processing, (2013).

[2] X. J. Sun and G. M. Yan, "Self-tuning weighted measurement fusion white noise deconvolution estimator and its convergence analysis", Digital Signal Processing, (2013).

[3] Z. L. Deng, "Information Fusion Estimation Theory and its Application", Harbin, Science Press, (2012).

[4] P. J. Dua, S. C. Liu, J. S. Xia and Y. D. Zhao, "Information fusion techniques for change detection from multi-temporal remote sensing images", Information Fusion, vol. 14, no. 1, (2013), pp. 19-27.

[5] B. Khaleghi, A. Khamis, F. O. Karray and S. N. Razavi, "Multisensor data fusion: A review of the state-of-the-art", Information Fusion, vol. 14, no. 1, (2013), pp. 28-44.

[6] M. Oussalah, Z. Messaoudi and A. Ouldali, "Track-to-Track Measurement Fusion Architectures and Correlation Analysis", Journal of Universal Computer Science, vol. 16, no. 1, (2010), pp. 37-61.

[7] P. Cui, H. S. Zhang, H. X. Wang and J. E. Feng, "Globally optimal real-time distributed fusion of multichannel observation systems", Control Theory \& Applications, vol. 8, no. 6, (2014), pp.384- 388.

[8] M. B. Trawicki and M. T. Johnson, "Distributed multichannel speech enhancement with minimum mean-square error short-time spectral amplitude, log-spectral amplitude, and spectral phase estimation", Signal Processing, (2012).

[9] C. J. Ran and Z. L. Deng, "Self-tuning distributed measurement fusion Kalman estimator for the multichannel ARMA signal”, Signal Processing, (2011).

[10] G. Hao, X. F. Ye and T. Chen, "Weighted measurement fusion algorithm for nonlinear unscented Kalman filter", Control Theory \& Applications, vol. 28, no. 6, (2011), pp. 753-758.

[11] J. X. Feng, Z. D. Wang and M. Zeng, "Distributed weighted robust Kalman filter fusion for uncertain systems with auto correlated and cross-correlated noises", Information Fusion, vol. 14, no. 1, (2013), pp. 78-86.

[12] Z. L. Deng, P. Zhang, W. J. Qi, Y. Gao and J. F. Liu, "The accuracy comparison of multisensor covariance intersection fuser and three weighting fusers", Information Sciences, vol. 14, no. 2, (2013), pp. 177-185.

[13] W. J. Qi, P. Zhang and Z. L. Deng, "Two-level robust sequential covariance intersection fusion Kalman predictors over clustering sensor networks with uncertain noise variances", International Journal of Sensor Networks, vol. 14, no. 4, (2013), pp. 251-261.

[14] W. J. Qi, P. Zhang and Z. L. Deng, "Covariance Intersection Fusion Kalman Estimators for MultiSensor System with Colored Measurement Noises", Research Journal of Applied Sciences, Engineering and Technology, vol. 6, no. 10, (2013), pp. 1872-1878.

[15] S. L. Sun and Z. L. Deng, "Multi-sensor optimal information fusion Kalman filter", Automatica, vol. 40, no. 6, (2004). pp.1017-1023.

[16] L. J. Chen, P. O. Arambel and R. K. Mehra, "Estimation Under Unknown Correlation: Covariance Intersection Revisited", IEEE Transactions on Automatic Control, vol. 47, no. 11, (2002), pp.1879-1882.

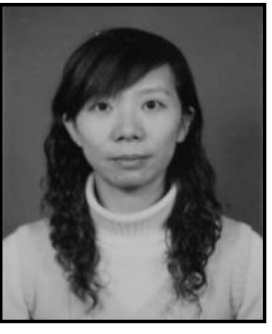

Yun Li, she is an associate professor at Harbin University of Commerce now. She obtained her bachelor's degree and master's degree in Heilongjiang University. Her major researches are state estimation, information fusion, etc.

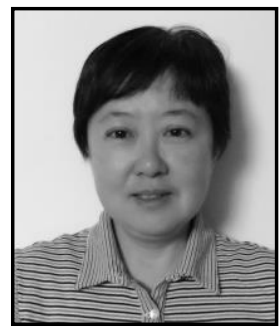

Ming Zhao, she is an associate professor at Harbin University of Commerce now. She obtained her bachelor's degree and master's degree in Harbin Engineering University. Her major researches are pattern recognition, information fusion, etc. 


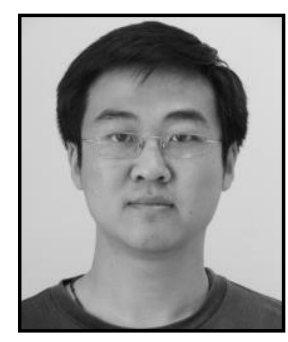

Gang Hao, he is an associate professor at Heilongjiang University now. He obtained his bachelor's degree and master's degree in Heilongjiang University, and obtained his Ph.D. in Harbin Engineering University. His major researches are state estimation, information fusion, etc. 
International Journal of Control and Automation Vol. 8, No.4 (2015) 